\title{
Transient Disturbance in a Half-Space under Generalized Magneto-Thermoelasticity with Internal Heat Source
}

\author{
Mohamed I.A. Othman ${ }^{a, *}, \mathrm{KH}$ Lotfy $^{b}$ And R.M. FArouK ${ }^{c}$ \\ ${ }^{a}$ Department of Mathematics, Faculty of Science, Zagazig University \\ P.O. Box 44519, Zagazig, Egypt \\ ${ }^{b}$ Computer Science Department, Faculty of Science, Al-Dawadmi, King Saud University \\ P.O. Box 18, Al-Dawadmi 11911, Kingdom of Saudi Arabia \\ (Received March 18, 2009; revised version May 18, 2009; in final form June 15, 2009)
}

\begin{abstract}
The transient waves caused by a line heat source with a uniform velocity inside isotropic homogeneous thermoelastic perfectly conducting half-space permeated into a uniform magnetic field are studied. The formulation is applied under three theories of generalized thermoelasticity: Lord-Shulman theory with one relaxation time, Green-Lindsay theory with two relaxation times, as well as the classical dynamical coupled theory. The problem is reduced to the solution of three differential equations by introducing the elastic and thermoelastic potentials. The normal mode analysis is used to obtain the expression for the temperature, displacement components and the thermal stresses. Numerical results are given and illustrated graphically. Comparisons are made with the results predicted by the three theories in the presence and absence of magnetic field and the internal heat source.
\end{abstract}

PACS numbers: 46.25.Hf

\section{Introduction}

The generalized thermoelasticity theories admit so-called second-sound effects, that is, which predict only finite velocity of propagation for heat field. At present, there are two different theories of the generalized thermoelasticity; the first was developed by Lord and Shulman (L-S) [1] who obtained wave-type heat conduction by postulating a new law of heat conduction to replace the classical Fourier law. This new law contains the heat flux vector as well as its time derivative. It contains also a new constant that acts as a relaxation time. Lord and Shulman's theory with a thermal relaxation time has been used by several authors including Puri [2] and Nayfeh and Nemat-Nasser [3] to study plane thermoelastic waves in non-rotating infinite media. Surface waves have been also studied by Agarwal [4] in the generalized thermoelasticity. By using the state space approach, Ezzat and Othman [5] have studied a two-dimensional magneto-thermoelasticity plane waves with thermal relaxation in a non-rotating medium of perfect conductivity, a two-dimensional electro-magneto-thermoelastic problem was dealt with for a finitely conducting half-space by Sherief and Helmy [6]. Othman [7] used the normal mode analysis to study two-dimensional problems

* corresponding author; e-mail: m_i_othman@yahoo.com of generalized thermoelasticity with one relaxation time with the modulus of elasticity dependent on the reference temperature for non-rotating and rotating medium, respectively. Ezzat et al. [8] applied the normal mode analysis to a two-dimensional electro-magneto-thermoelastic plane wave's problem of a medium of perfect conductivity with one relaxation time.

The second was developed by Green and Lindsay (G-L) [9]. This theory contains two constants that act as relaxation times and modifies all the equations of the coupled theory not the heat conduction equation only. The two theories both ensure finite speeds of propagation for heat wave. Using the Green-Lindsay theory, Agarwal [10] studied respectively thermoelastic and magneto-thermoelastic plane wave propagation in an infinite non-rotation medium. In a paper by Schoenberg and Censor [11], the propagation of plane harmonic waves in a rotating elastic medium without a thermal field has been studied. It was shown there that the rotation causes the elastic medium to be depressive and anisotropic.

Great attention has been devoted to the study of electro-magneto-thermoelastic coupled problems based on the generalized thermoelastic theories. In the context of Green and Lindsay theory, Roy Choudhuri and Mukhopadhyay [12] studied the propagation of electro-magneto-thermoelastic harmonic waves in an unbounded isotropic conducting medium permeating uniform field when the entire medium rotates with a uni- 
form angular velocity; Ezzat and Othman [13] applied the normal mode analysis to a problem of two-dimensional electro-magneto-thermoelastic plane waves with two relaxation times in a medium of perfect conductivity. Dhaliwal and Rokne [14] have solved a thermal shock problem. Recently Othman [15] considered a problem of plane wave propagation in a rotating medium in generalized thermoelasticity with two relaxation times.

Heat source acting in an elastic body is widely used in engineering involving materials processing, case hardening and boiling nucleation etc. This kind of problem is very interesting in mathematics and important in physics. Sarbani and Amitava Chakravorty [16] studied the transient disturbances in half-space due to moving internal heat source under L-S model and obtained the solution for the displacements in the transform domain. Under G-L theory, Chandrasekharaiah and Srinath [17] have studied the cases of both continuous and impulsive point heat source in an unbounded body and obtained small time solutions with the aid of Laplace transforms. Chandrasekharaiah and Murthy [18] have studied cylindrical waves due to a continuous line heat source in an unbounded body and have obtained small-time solutions by employing the Laplace and Hankel transform. Hetnarski and Ignaczak [19] have studied a plane heat sources in half-space in great detail and have presented closed form solutions.

In the present work we shall formulate the magneto-thermoelastic coupled two-dimensional problem of a thermally and perfect conducting half-space solid in the presence of moving internal heat source. The normal mode method is used to obtain the exact expressions for the considered variables. The distributions of the considered variables are represented graphically. A comparison is carried out between the temperature, stresses and displacements as calculated from the generalized thermo-elasticity L-S, G-L and classical dynamical (CD) theories for the propagation of waves in semi-infinite elastic solids in present and absent magnetic field and the internal heat source.

\section{Formulation of the problem}

Let us consider a linear, homogeneous and isotropic thermoelastic continuum occupying the region $G$ given by $G=\{(x, y, z) \mid y \geq-L, 0 \leq x,-\infty<z<\infty\}$, and the surface $y=-L$ which is stress free. The elastic medium is permeated into a uniform magnetic field with constant intensity $\boldsymbol{H}=\left(0,0, H_{0}\right)$ which is parallel to $z$-axis. A line source suddenly starts moving inside the medium at a depth $L$ below the free surface with a uniform velocity in the $x$-direction. The line source is parallel to the $z$-axis so that all quantities are independent of $z$, and the third component $w$ of the displacement vector vanishes. When all body forces are neglected the governing equations are:

(1) strain-displacement relations

$$
e_{i j}=\frac{1}{2}\left(u_{i, j}+u_{j, i}\right), \quad i, j=1,2 .
$$

where $u_{i}=(u, v, 0)$ are the components of displacement vector, $e_{i j}$ are the components of strain tensor.

(2) Stress displacement relation

$$
\sigma_{i j}=2 \mu e_{i j}+\lambda e \delta_{i j}-\gamma\left(1+\nu_{0} \frac{\partial}{\partial t}\right) T \delta_{i j} .
$$

(3) Heat conduction equation

$$
\begin{aligned}
& k T_{i i}=\rho C_{E}\left(n_{1}+\tau_{0} \frac{\partial}{\partial t}\right) \dot{T}+\gamma T_{0}\left(n_{1}+n_{0} \tau_{0} \frac{\partial}{\partial t}\right) \dot{e} \\
& -\left(n_{1}+n_{0} \tau_{0} \frac{\partial}{\partial t}\right) Q,
\end{aligned}
$$

where $n_{0}$ and $n_{1}$ are parameters, $Q$ is a moving internal heat source and $T_{0}$ is the reference temperature.

(4) Equation of motion

$$
\rho \ddot{u}_{i}=\sigma_{i j, j}+F_{i},
$$

where $F_{i}$ is the Lorentz force and is given in the form

$$
F_{i}=\mu_{0}(J \times H)_{i} .
$$

The variation of the magnetic and electric fields are perfectly conducting slowly moving medium and are given by Maxwell's equations

$$
\begin{aligned}
& \operatorname{curl} \boldsymbol{h}=\boldsymbol{J}+\dot{\boldsymbol{D}}, \\
& \operatorname{curl} \boldsymbol{E}=-\dot{\boldsymbol{B}}, \\
& \operatorname{div} \boldsymbol{B}=0, \quad \operatorname{div} \boldsymbol{D}=0, \quad \boldsymbol{B}=\mu_{0} \boldsymbol{H}, \quad \boldsymbol{D}=\varepsilon_{0} \boldsymbol{E}, \\
& \boldsymbol{E}=-\mu_{0}(\dot{\boldsymbol{u}} \times \boldsymbol{H}),
\end{aligned}
$$

where $\dot{\boldsymbol{u}}$ is the particle velocity of the medium, and the small effect of temperature gradient on $\boldsymbol{J}$ is also ignored. The dynamic displacement vector is actually measured from a steady-state deformed position and the deformation is supposed to be small.

The components of magnetic intensity vector in the medium are

$$
\begin{aligned}
& H_{1}=H_{2}=0, \quad H_{3}=\mu_{0}\left[H_{0}+h(x, z, t)\right], \\
& J_{1}=-H_{0} \frac{\partial e}{\partial y}+\mu_{0} H_{0} \varepsilon_{0} \ddot{v}, \quad J_{2}=H_{0} \frac{\partial e}{\partial x}-\mu_{0} H_{0} \varepsilon_{0} \ddot{u}, \\
& J_{3}=0 .
\end{aligned}
$$

Substituting Eqs. (6)-(10) into Eq. (5) we obtain

$$
\begin{aligned}
& F_{1}=\mu_{0} H_{0}^{2} \frac{\partial e}{\partial x}-\mu_{0}^{2} H_{0}^{2} \varepsilon_{0} \frac{\partial^{2} u}{\partial t^{2}}, \\
& F_{2}=\mu_{0} H_{0}^{2} \frac{\partial e}{\partial y}-\mu_{0}^{2} H_{0}^{2} \varepsilon_{0} \frac{\partial^{2} v}{\partial t^{2}}, \quad F_{3}=0,
\end{aligned}
$$

where

$$
\boldsymbol{h}=-H_{0}(0,0, e) \text {. }
$$

Substituting Eq. (2) and (11) into Eq. (4) we get

$$
\begin{aligned}
& \rho \ddot{u}_{i}=(\lambda+\mu) u_{j, j i} \delta_{i j}+\mu u_{i, j j}-\gamma\left(1+\nu_{0} \frac{\partial}{\partial t}\right) T_{, i} \delta_{i j} \\
& \quad+\mu_{0} H_{0}^{2} e_{, i}-\mu_{0}^{2} H_{0}^{2} \varepsilon_{0} \ddot{u}_{i} .
\end{aligned}
$$

Equations (3) and (13) are the field equations of the generalized linear magneto-thermoelasticity, applicable to the coupled theory and two generalizations, as follows:

(i) The equations of the coupled magneto-thermoelasticity CD theory due to the moving internal heat source, when 
$\nu_{0}=\tau_{0}=0, \quad n_{0}=0, \quad n_{1}=1$.

Equations (3) and (13) have the form

$$
\begin{aligned}
& \rho \ddot{u}_{i}=(\lambda+\mu) u_{j, j i} \delta_{i j}+\mu u_{i, j j}-\gamma T_{, i} \delta_{i j}+\mu_{0} H_{0}^{2} e_{, i} \\
& \quad-\mu_{0}^{2} H_{0}^{2} \varepsilon_{0} \ddot{u}_{i}, \\
& k T_{, i i}=\rho C_{E} \dot{T}+\gamma T_{0} \dot{e}-Q .
\end{aligned}
$$

(ii) Lord and Shulman's theory due to internal heat source, when

$$
\nu_{0}=0, \quad n_{0}=n_{1}=1, \quad \tau_{0}>0 .
$$

Equation (13) is the same as Eq. (15) and Eq. (3) has the form

$$
k T_{, i i}=\left(1+\tau_{0} \frac{\partial}{\partial t}\right)\left(\rho C_{E} \dot{T}+\gamma T_{0} \dot{e}-Q\right) .
$$

(iii) Green and Lindsay's theory due to internal heat source, when

$$
n_{0}=0, \quad n_{1}=1, \quad \nu_{0} \geq \tau_{0}>0 .
$$

Equation (13) remains without change and Eq. (3) has the form

$$
k T_{, i i}=\rho C_{E}\left(1+\tau_{0} \frac{\partial}{\partial t}\right) \dot{T}+\gamma T_{0} \dot{e}-Q .
$$

(iv) The correspondent equations for the generalized thermoelasticity without magnetic field due to moving internal heat source results from the above mentioned cases by taking $H_{0}=0$.

Introducing the scalar and vector potentials $\varphi, \psi$ defined by

$$
u=\varphi_{, x}+\psi_{, y}, \quad v=\varphi_{, y}-\psi_{, x},
$$

where

$$
\varphi=\varphi(x, y, t), \quad \text { and } \quad \psi=\psi(x, y, t) .
$$

Taking divergence and curl of equation of motion we obtain

$$
\begin{aligned}
& \nabla^{2} \varphi-\frac{\alpha}{\beta^{2}} \ddot{\varphi}=m_{1}\left(1+\nu_{0} \frac{\partial}{\partial t}\right) T, \\
& \nabla^{2} \psi=\frac{\alpha}{C_{T}^{2}} \ddot{\psi} .
\end{aligned}
$$

$>$ From Eq. (3) we can obtain

$$
\begin{aligned}
k & \nabla^{2} T=\rho C_{E}\left(n_{1}+\tau_{0} \frac{\partial}{\partial t}\right) \dot{T} \\
& +\gamma T_{0}\left(n_{1}+n_{0} \tau_{0} \frac{\partial}{\partial t}\right) \nabla^{2} \dot{\varphi}-\left(n_{1}+n_{0} \tau_{0} \frac{\partial}{\partial t}\right) Q
\end{aligned}
$$

where

$$
\begin{aligned}
& \alpha=1+C_{A}^{2} / c^{2}, \quad C_{L}^{2}=(\lambda+2 \mu) / \rho, \\
& \beta^{2}=C_{L}^{2}+C_{A}^{2}, \quad C_{T}^{2}=\mu / \rho, \quad \nabla^{2}=\frac{\partial^{2}}{\partial x^{2}}+\frac{\partial^{2}}{\partial y^{2}} .
\end{aligned}
$$

In order to use non-dimensional of Eqs. (23)-(25), let us define the following set of dimensionless variables:

$$
\begin{aligned}
& \bar{x}_{i}=\frac{x_{i}}{C_{T} \omega^{*}}, \quad \bar{u}_{i}=\frac{u_{i}}{C_{T} \omega^{*}}, \quad \bar{\varphi}=\frac{\varphi}{\left(C_{T} \omega^{*}\right)^{2}}, \\
& \bar{\psi}=\frac{\psi}{\left(C_{T} \omega^{*}\right)^{2}}, \quad \overline{\tau_{0}}=\frac{\tau_{0}}{\omega^{*}}, \quad \bar{\nu}_{0}=\frac{\nu_{0}}{\omega^{*}}, \\
& \theta=m_{1} T, \quad \bar{\sigma}_{i j}=\frac{\sigma_{i j}}{\mu}, \quad \bar{h}=\frac{h}{H_{0}}, \quad \bar{Q}=\frac{m_{1} \omega^{*} Q}{\rho c},
\end{aligned}
$$

$$
\omega^{*}=\frac{K}{\rho C_{E} C_{T}^{2}}, \quad i=1,2,
$$

where $m_{1}=\frac{\gamma}{\rho \beta^{2}}$ (dropping the over bar for convenience).

Equations (23)-(25) become

$$
\begin{aligned}
& \nabla^{2} \varphi-\alpha \beta_{1}^{2} \ddot{\varphi}=\left(1+\nu_{0} \frac{\partial}{\partial t}\right) \theta, \\
& \nabla^{2} \psi=\alpha \ddot{\psi}, \\
& \nabla^{2} \theta=\left(n_{1}+\tau_{0} \frac{\partial}{\partial t}\right) \dot{\theta}+\bar{\varepsilon}\left(n_{1}+n_{0} \tau_{0} \frac{\partial}{\partial t}\right) \nabla^{2} \dot{\varphi} \\
& -\left(n_{1}+n_{0} \tau_{0} \frac{\partial}{\partial t}\right) Q,
\end{aligned}
$$

where $\beta_{1}^{2}=\frac{C_{T}^{2}}{\beta^{2}}, \bar{\varepsilon}=\frac{\gamma T_{0} m_{1}}{\rho C_{E}}$ and $\bar{\varepsilon}$ is the coupling parameter.

The constitutive equations reduce to

$$
\begin{aligned}
\sigma_{x x} & =\left(\alpha_{1}^{2}-2\right) e+2 u_{, x}-\beta_{1}^{*^{2}}\left(1+\nu_{0} \frac{\partial}{\partial t}\right) \theta, \\
\sigma_{y y} & =\left(\alpha_{1}^{2}-2\right) e+2 v_{, y}-\beta_{1}^{*^{2}}\left(1+\nu_{0} \frac{\partial}{\partial t}\right) \theta, \\
\sigma_{x y} & =u_{, y}+v_{, x},
\end{aligned}
$$

where

$$
\alpha_{1}^{2}=\frac{\lambda}{\mu}+2 \quad \text { and } \quad \beta_{1}^{*^{2}}=\frac{\gamma}{m_{1} \mu} .
$$

\section{Normal mode analysis}

The solution of the considered physical variables can be decomposing in terms of normal modes analysis as following from:

$$
\begin{aligned}
& {\left[\varphi, \psi, \theta, \sigma_{i j}\right](x, y, t)=\left[\varphi^{*}(x), \psi^{*}(x), \theta^{*}(x), \sigma_{i j}^{*}(x)\right]} \\
& \quad \times \exp (\omega t+\mathrm{i} a y), \\
& Q=Q^{*} \exp (\omega t+\mathrm{i} a y), \quad Q^{*}=Q_{0} v_{0},
\end{aligned}
$$

where $\omega$ is the (complex) time constant, $\mathrm{i}=\sqrt{-1}$, $a$ is the wave number in the $y$-direction and $u^{*}(x), w^{*}(x), \varphi^{*}(x), \psi^{*}(x), \theta^{*}(x)$ and $\sigma_{i j}^{*}(x)$ are the amplitude of the field quantities, $v_{0}$ is the velocity of moving internal heat source and $Q_{0}$ is the magnitude of the internal heat source.

Using Eqs. (34) and (35), Eqs. (27)-(29) become respectively

$$
\begin{aligned}
& \left(D^{2}-a_{1}^{2}\right) \varphi^{*}(x)=\left(1+\nu_{0} \omega\right) \theta^{*}(x), \\
& \left(D^{2}-m^{2}\right) \psi^{*}(x)=0, \\
& \left(D^{2}-a_{4}\right) \theta^{*}(x)=a_{3}\left(D^{2}-a^{2}\right) \varphi^{*}(x)-A Q_{0},
\end{aligned}
$$

where $D=\frac{\mathrm{d}}{\mathrm{d} x}, A=A^{\prime} v_{0}, A^{\prime}=n_{1}+n_{0} \tau_{0} \omega$,

$$
\begin{aligned}
& a_{1}=a^{2}+\alpha \beta_{1}^{2} \omega^{2}, \\
& a_{2}=\omega\left(n_{1}+\tau_{0} \omega\right), \\
& a_{3}=\omega \bar{\varepsilon} A^{\prime}, \\
& a_{4}=a^{2}+a_{2}, \\
& m^{2}=a^{2}+\alpha \omega^{2} .
\end{aligned}
$$

Eliminating $\theta^{*}(x)$ between Eqs. (36) and (38), we get 
the following fourth order ordinary differential equation satisfied by $\varphi^{*}(x)$ :

$$
\left(D^{4}-b_{1} D^{2}+b_{2}\right) \varphi^{*}(x)=-A\left(1+\nu_{0} \omega\right) Q_{0},
$$

where

$$
\begin{aligned}
& b_{1}=a_{1}+\left(1+\nu_{0} \omega\right) a_{3}+a_{4}, \\
& b_{2}=a_{1} a_{4}+\left(1+\nu_{0} \omega\right) a_{3} a^{2} .
\end{aligned}
$$

Equation (44) can be factorized as

$$
\left(D^{2}-k_{1}^{2}\right)\left(D^{2}-k_{2}^{2}\right) \varphi^{*}(x)=-A\left(1+\nu_{0} \omega\right) Q_{0},
$$

where $k_{j}^{2}, j=1,2$ are the roots of the following characteristic equation:

$$
k^{4}-b_{1} k^{2}+b_{2}=0 .
$$

The solution of Eq. (44) is given by

$$
\varphi^{*}(x)=\sum_{j=1}^{2} R_{j}(a, \omega) \mathrm{e}^{-k_{j} x}-\frac{A\left(1+\nu_{0} \omega\right) Q_{0}}{b_{2}} .
$$

In a similar manner, we get

$$
\begin{aligned}
& \theta^{*}(x)=\sum_{j=1}^{2}\left[\left(k_{j}^{2}-a_{1}\right) /\left(1+\nu_{0} \omega\right)\right] R_{j}(a, \omega) \mathrm{e}^{-k_{j} x} \\
& +\frac{A a_{1} Q_{0}}{b_{2}}
\end{aligned}
$$

where $R_{j}(a, \omega)$ are parameters depending on $a$ and $\omega$.

The solution of Eq. (37) can be written as

$$
\psi^{*}(x)=R_{3} \mathrm{e}^{-m x},
$$

since

$$
\begin{aligned}
& u^{*}(x)=D \varphi^{*}+\mathrm{i} a \psi^{*}, \\
& v^{*}(x)=\mathrm{i} a \varphi^{*}-D \psi^{*}, \\
& e^{*}(x)=D u^{*}+\mathrm{i} a v^{*} .
\end{aligned}
$$

Using Eqs. (49) and (51), in order to obtain the amplitude of the displacement components $u$ and $v$, which are bounded as $x \rightarrow \infty$, then Eqs. (52) and (53) become

$$
\begin{aligned}
& u^{*}(x)=-\sum_{j=1}^{2} R_{j}(a, \omega) k_{j} \mathrm{e}^{-k_{j} x}+\mathrm{i} a R_{3} \mathrm{e}^{-m x}, \\
& v^{*}(x)=\mathrm{i} a\left[\sum_{j=1}^{2} R_{j}(a, \omega) \mathrm{e}^{-k_{j} x}-\frac{A\left(1+\nu_{0} \omega\right) Q_{0}}{b_{2}}\right] \\
& +m R_{3} \mathrm{e}^{-m x} .
\end{aligned}
$$

The stress component is in the form

$$
\begin{aligned}
& \sigma_{x x}^{*}=\sum_{j=1}^{2}\left[k_{j}^{2}\left(\alpha_{1}^{2}-\beta_{1}^{*^{2}}\right)-\left(\alpha_{1}^{2}-2\right) a^{2}+\beta_{1}^{*^{2}} a_{1}\right] \\
& \quad \times R_{j} \mathrm{e}^{-k_{j} x}-2 \mathrm{i} a m R_{3} \mathrm{e}^{-m x} \\
& \quad+\left[\left(\alpha_{1}^{2}-2\right) a^{2}-\beta_{1}^{*^{2}} a_{1}\right] \frac{A\left(1+\nu_{0} \omega\right) Q_{0}}{b_{2}}, \\
& \sigma_{y y}^{*}=\sum_{j=1}^{2}\left[k_{j}^{2}\left(\alpha_{1}^{2}-\beta_{1}^{*^{2}}-2\right)-\alpha_{1}^{2} a^{2}+\beta_{1}^{*^{2}} a_{1}\right] \\
& \quad \times R_{j} \mathrm{e}^{-k_{j} x}+2 \mathrm{i} a m R_{3} \mathrm{e}^{-m x} \\
& \quad+\left(a^{2} \alpha_{1}^{2}-\beta_{1}^{*^{2}} a_{1}\right) \frac{A\left(1+\nu_{0} \omega\right) Q_{0}}{b_{2}},
\end{aligned}
$$

$$
\sigma_{x y}^{*}=-2 \mathrm{i} a \sum_{j=1}^{2} R_{j} k_{j} \mathrm{e}^{-k_{j} x}-\left(a^{2}+m^{2}\right) R_{3} \mathrm{e}^{-m x} .
$$

\section{Application}

We consider a homogeneous isotropic thermoelastic solid occupying the region $G$ given by $G=$ $\{(x, y, z) \mid y \geq-L, 0 \leq x,-\infty<z<\infty\}$.

In the physical problem, we should suppress the positive exponentials that are unbounded at infinity. The constants $R_{1}, R_{2} R_{3}$ have to be chosen such that the boundary conditions on the surface at $x=0$ take the form

$$
\begin{aligned}
\frac{\partial \theta}{\partial x} & =0, \\
\sigma_{x y} & =0, \\
\sigma_{x x} & =p_{0}(y, t),
\end{aligned}
$$

where $p_{0}$ is a given function of $y$ and $t$, with the initial conditions

$$
u=0 \text { and } v=0 \text { at } t=0 .
$$

Equations (60)-(62a) in the normal mode form together with Eqs. (43), (57) and (60) respectively, give

$$
\begin{aligned}
& L_{1} R_{1}+L_{2} R_{2}=0, \\
& M_{1} R_{1}+M_{2} R_{2}+M_{3} R_{3}=0, \\
& N_{1} R_{1}+N_{2} R_{2}+N_{3} R_{3}=p^{\prime} .
\end{aligned}
$$

Equations (63)-(65) can be solved for the three unknowns $R_{1}, R_{2}$ and $R_{3}$.

The solution of these equations can be written as

$$
\begin{aligned}
& R_{1}=\frac{\Delta_{1}}{\Delta}, \\
& R_{2}=\frac{\Delta_{2}}{\Delta}, \\
& R_{3}=\frac{\Delta_{3}}{\Delta} .
\end{aligned}
$$

where

$$
\begin{aligned}
& \Delta_{1}=-L_{2} M_{3} p^{\prime}, \\
& \Delta_{2}=-L_{1} M_{3} p^{\prime} \\
& \Delta_{3}=\left(L_{1} M_{2}-L_{2} M_{1}\right) p^{\prime}, \\
& \Delta=L_{1} \lambda_{1}-L_{2} \lambda_{2} \text {, } \\
& L_{j}=k_{j}\left[\left(k_{j}^{2}-a_{1}\right) /\left(1+\nu_{0} \omega\right)\right], \quad j=1,2, \\
& M_{j}=2 \mathrm{i} a k_{j}, \quad j=1,2, \\
& M_{3}=a^{2}+m^{2} \text {, } \\
& N_{j}=k_{j}^{2}\left(\alpha_{1}^{2}-\beta_{1}^{*^{2}}\right)-\left(\alpha_{1}^{2}-2\right) a^{2}+\beta_{1}^{*^{2}} a_{1}, \\
& j=1,2, \\
& N_{3}=-2 \mathrm{i} a m \text {, } \\
& p^{\prime}=p_{0}-\left[a^{2}\left(\alpha_{1}^{2}-2\right)-\beta_{1}^{*^{2}} a_{1}\right] \frac{A\left(1+\nu_{0} \omega\right) Q_{0}}{b_{2}},
\end{aligned}
$$




$$
\begin{aligned}
& \lambda_{1}=M_{2} N_{3}-M_{3} N_{2}, \\
& \lambda_{2}=M_{1} N_{3}-M_{3} N_{1} .
\end{aligned}
$$

\section{Numerical results and discussion}

In order to illustrate the theoretical results obtained in preceding section and to compare these in the context of various theories of thermoelasticity, we now present some numerical results. In the calculation process, we consider the material medium as that of copper. Since $\omega$ is the complex time constant then we take $\omega=\omega_{0}+\mathrm{i} \zeta$. The other constants of the problem are taken as $\omega_{0}=2 ; \zeta=1$; $p^{*}=100 ; a=5 ; T_{0}=293 \mathrm{~K}, \lambda=7.55 \times 10^{10} \mathrm{~kg} \mathrm{~m}^{-1} \mathrm{~s}^{-2}$, $\mu=3.86 \times 10^{10} \mathrm{~kg} \mathrm{~m}^{-1} \mathrm{~s}^{-2}, C_{l}=3.845 \times 10^{2} \mathrm{~m}^{2} \mathrm{~K}^{-1} \mathrm{~s}^{-2}$, $\alpha_{\mathrm{t}}=17.87 \times 10^{-6} \mathrm{~K}^{-1}, \rho=8.96 \times 10^{3} \mathrm{~kg} \mathrm{~m}^{-3}, \mu_{0}=$ $4 \pi \times 10^{-7}$.

Figures 1-12 show six curves predicted by different theories of thermoelasticity. In these figures, the solid lines represent the $\mathrm{CD}$ theory, dashed lines represent the L-S theory and the dotted lines represent the G-L theory. At the boundary surface, $y=-5$, the distributions of displacement components $u$ and $v$ versus time are shown in Figs. 1 and 3 under three theories at the nondimensional quantities $Q_{0}=10, v_{0}=2$ and $\alpha=1.8$, i.e. in presence of moving internal heat source and magnetic field. Figures 1 and 3 depict the distributions of displacement components $u$ and $v$ versus $x$ at the boundary for different values of time (e.g. $t=0.1, t=0.4$ ); we can see that the displacement component $v$ always starts from the zero value and terminates at the zero value. Also we can see that the displacement decreases monotonically with $x$ as increasing the time $t$. Figure 1 shows that in all three theories CD, L-S and G-L the values of displacement component $u$ for $t=0.4$ are large compared with those for $t=0.1$, the values of displacement component $u$ decrease in the ranges $0 \leq x \leq 0.4$ and $3 \leq x \leq 5.5$, the values of displacement component $u$ increase in the ranges $0.4 \leq x \leq 3$ and $5.5 \leq x \leq 8$ for $t=0.4$, but the values of displacement component $u$ decrease in the ranges $0 \leq x \leq 0.8$ and $3.2 \leq x \leq 6$ for $t=0.1$, then increase otherwise in the range $0 \leq x \leq 10$, while converge to zero with increasing the distance $x$ at $x \geq 10$. Figure 3 shows that in all three theories the values of displacement component $v$ for $t=0.1$ are large compared to those for $t=0.4$, the values of displacement component $v$ decrease with increasing the distance $x$ and converge to zero with increasing the distance $x$ for $x \geq 10$.

Figures 2 and 4 show the distributions of displacement components $u$ and $v$ versus $x$ at the boundary at $t=0.1, v=2$ and the internal heat source $Q_{0}=10$ for different values of magnetic field ( $\alpha=1$ without magnetic field and $\alpha=1.8$ with magnetic field). It should be observed that the displacement component $v$ always starts from the zero value in the range $0 \leq x \leq 10$ and terminates at the zero value, and decreases monotonically with $x$ as increasing the magnetic field. Also we can observe that the displacement component $u$ increases monotonically with $x$ as increasing the magnetic field. The two

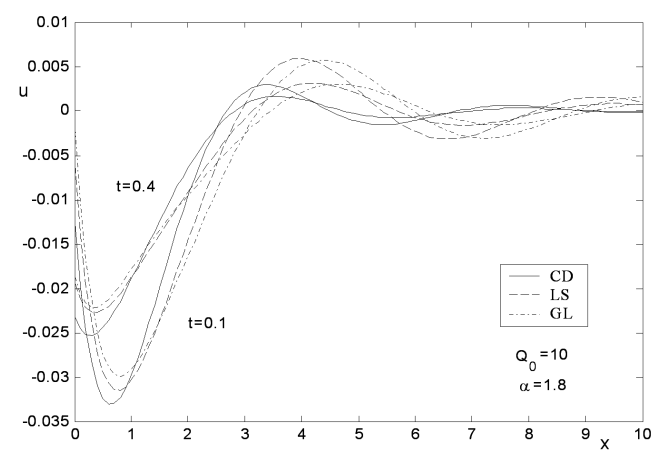

Fig. 1. Displacement distribution $u$ versus $x$ with different times.

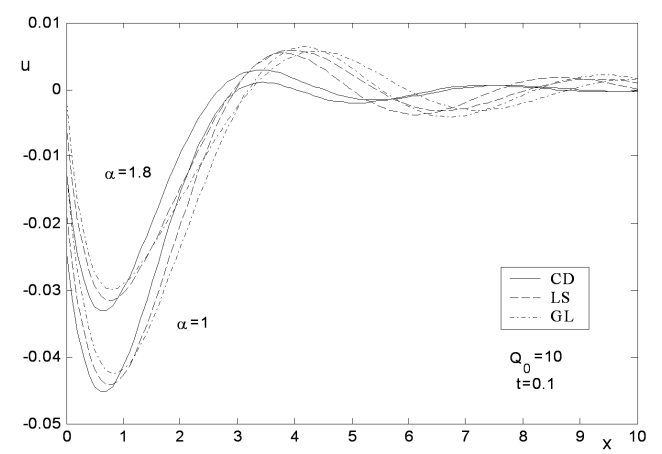

Fig. 2. Displacement distribution $u$ versus $x$ with different external magnetic field.

figures show that the relaxation times have salient effect to the distribution of displacement components $u$ and $v$ at small time range. Figure 2 depicts that the displacement component $u$ under L-S theory is greater than that under the CD-theory while it is smaller than that under the G-L theory. With increase of the magnetic field, the relaxation time has a decreasing effect.

Behavior of temperature in all three theories CD, L-S and G-L for the two different values of $t$ is similar, as shown in Fig. 5. Values of the temperature for $t=0.4$

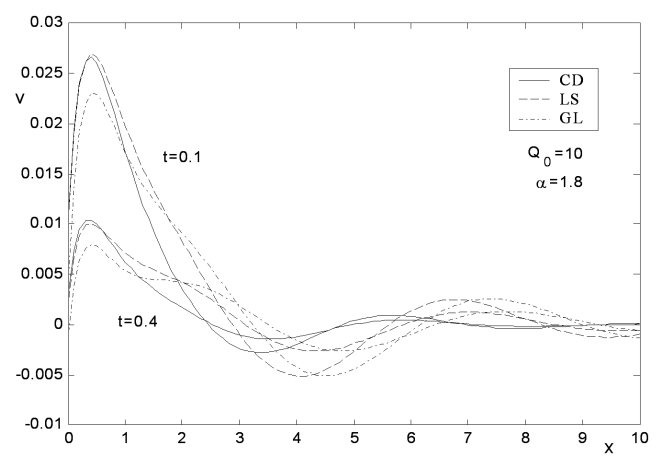

Fig. 3. Displacement distribution $v$ versus $x$ with different times. 


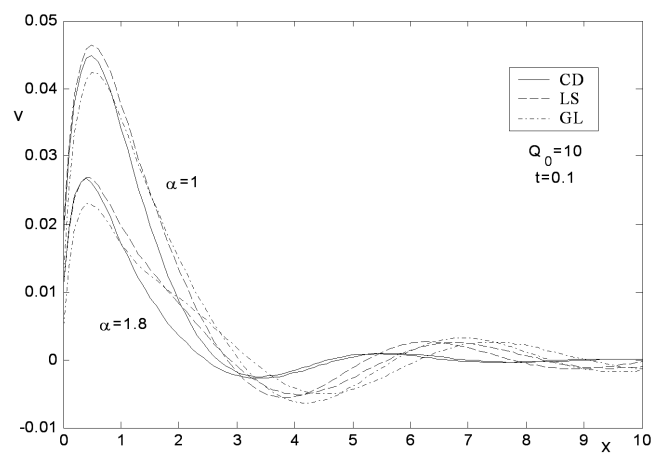

Fig. 4. Displacement distribution $v$ versus $x$ with different external magnetic field.

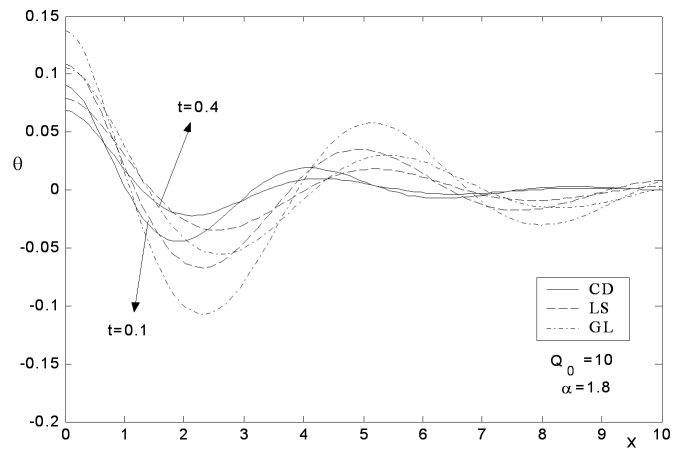

Fig. 5. Temperature distribution $\theta$ versus $x$ with different times.

are small compared to those for $t=0.1$ in the range $0 \leq x \leq 0.7$ for $\mathrm{CD}$, in the range $0 \leq x \leq 0.8$ for $\mathrm{L}-\mathrm{S}$ and in the range $0 \leq x \leq 0.9$ for $\mathrm{G}-\mathrm{L}$, but in the range $0.7 \leq x \leq 2.9$ for $\mathrm{CD}$, in the range $0.8 \leq x \leq 3.7$ for L-S and in the range $0.9 \leq x \leq 3.5$ for G-L are large, while values are the same for the three theories at $x>9$. Behavior of temperature in all three theories CD, L-S and $\mathrm{G}-\mathrm{L}$ for the two different values of magnetic field $(\alpha=1$ without magnetic field and $\alpha=1.8$ with magnetic field) is similar, as shown in Fig. 6. Values of temperature for

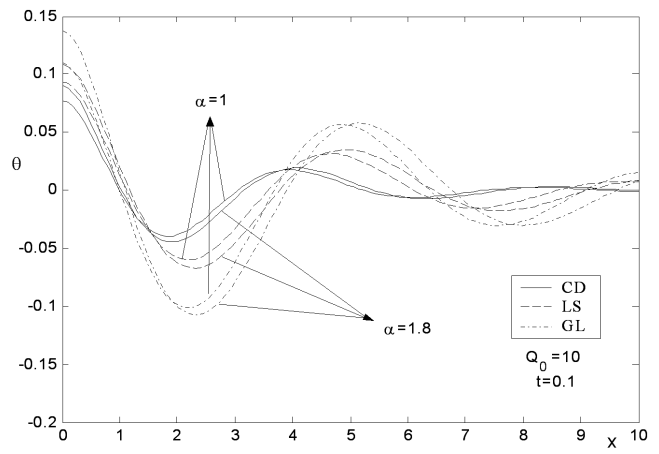

Fig. 6. Temperature distribution $\theta$ versus $x$ with different external magnetic field.

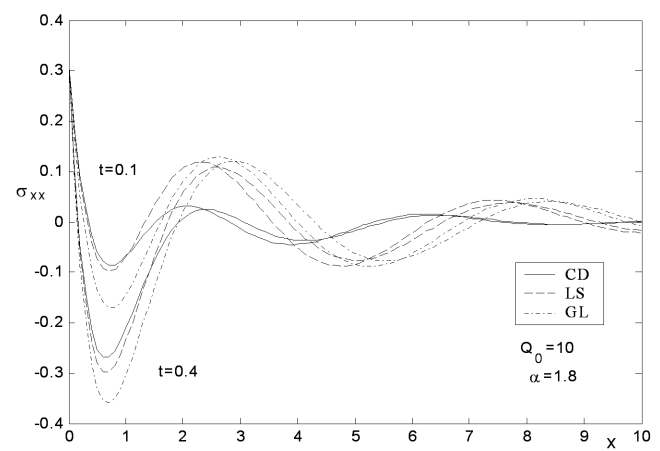

Fig. 7. Stress distribution $\sigma_{x x}$ versus $x$ with different times.

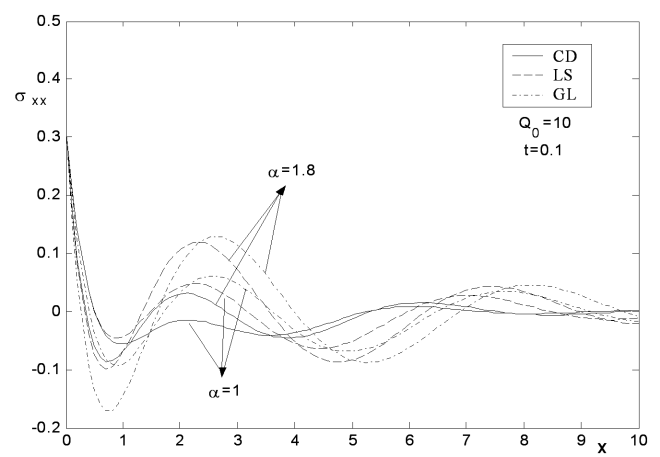

Fig. 8. Stress distribution $\sigma_{x x}$ versus $x$ with different external magnetic field.

$\alpha=1$ are small compared to those for $\alpha=1.8$ in the range $0 \leq x \leq 1.4$ for $\mathrm{CD}$, in the range $0 \leq x \leq 1.6$ for L-S and in the range $0 \leq x \leq 1.8$ for G- $\overline{\mathrm{L}}$, but in the range $1.4 \leq x \leq 3.8$ for $\overline{C D}$, in the range $1.6 \leq x \leq 4.6$ for $\mathrm{L}-\mathrm{S}$ and in the range $1.8 \leq x \leq 5$ for $\mathrm{G}$-L are large and then small, while values are the same for the three theories at $x>9$.

Behavior of normal stress $\sigma_{x x}$ in all three theories CD, L-S and G-L for $t=0.4$ are small compared to those for $t=0.1$ in the range $0 \leq x \leq 2.5$; large in the range $2.4 \leq$

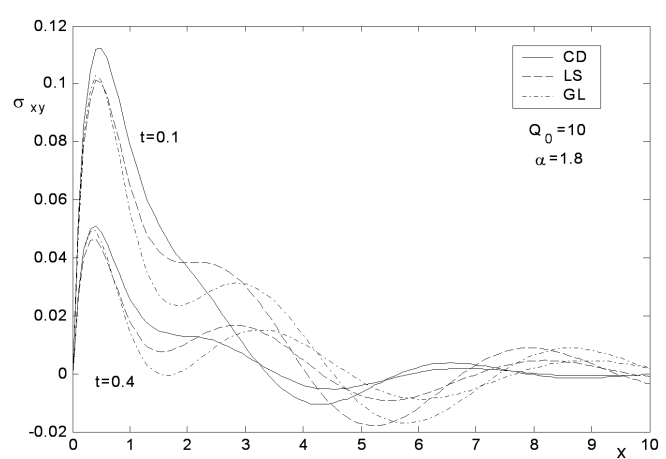

Fig. 9. Stress distribution $\sigma_{x y}$ versus $x$ with different times. 


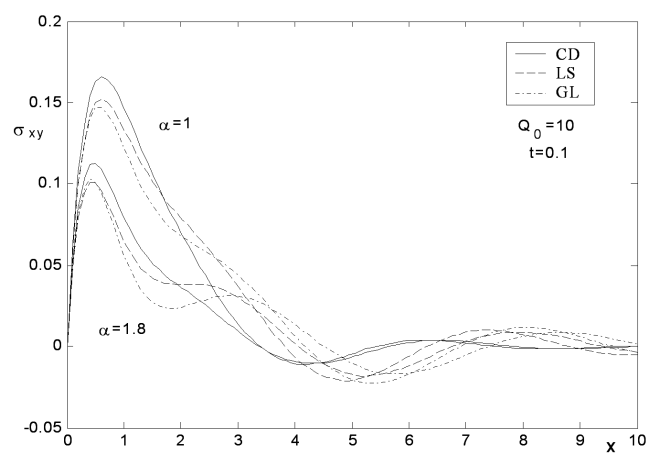

Fig. 10. Stress distribution $\sigma_{x y}$ versus $x$ with different external magnetic field.

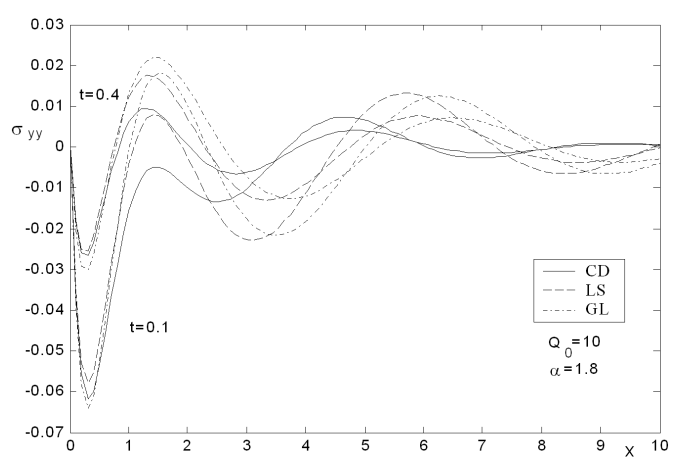

Fig. 11. Stress distribution $\sigma_{y y}$ versus $x$ with different times.

$x \leq 4.8$, while values are the same for the three theories at $x>10$, as depicted in Fig. 7. Behavior of normal stress $\sigma_{x x}$ in all three theories CD, L-S and G-L for the two different values of magnetic field $(\alpha=1$ without magnetic field and $\alpha=1.8$ with magnetic field) is similar, as shown in Fig. 8. Values of normal stress for $\alpha=1$ are large compared to those for $\alpha=1.8$ in the range $0 \leq x \leq 1.1$ for $\mathrm{CD}$, in the range $0 \leq x \leq 1.3$ for $\mathrm{L}-\mathrm{S}$ and in the range $0 \leq x \leq 1.6$ for $\mathrm{G}-\mathrm{L}$, but in the range $1.1 \leq x \leq 3.7$ for $\mathrm{CD}$, in the range $1.3 \leq x \leq 4.1$ for $\mathrm{L}-\mathrm{S}$

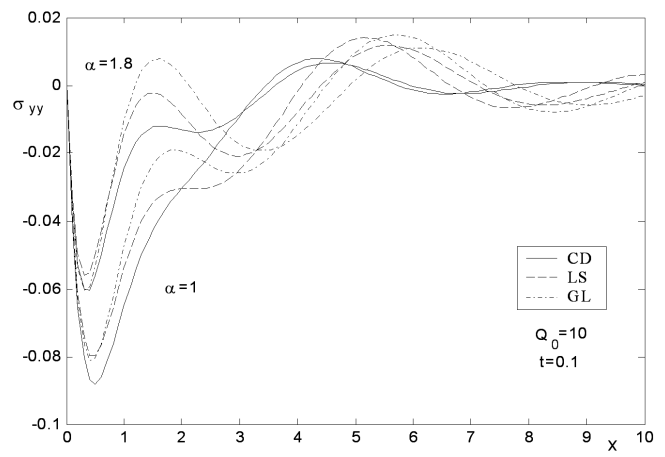

Fig. 12. Stress distribution $\sigma_{y y}$ versus $x$ with different external magnetic field. and in the range $1.6 \leq x \leq 4.7$ for G-L are small, while values are the same for the three theories at $x>9$.

Behaviors of tangential coupled stress in all three theories CD, L-S and G-L are for the two different values of magnetic field in the range $0 \leq x \leq 3.2$ for $\mathrm{CD}$, in the range $0 \leq x \leq 3.8$ for $\mathrm{L}-\mathrm{S}$ and in the range $0 \leq x \leq 4.3$ for $\mathrm{G}-\mathrm{L}$, also in the range $5.4 \leq x \leq 7.5$ for $\mathrm{CD}, 6.5 \leq x \leq 9.2$ for L-S and $7.2 \leq x \leq 10$ for G-L, large otherwise, while values are the same for the three theories and converge to zero at $x>10$.

Behaviors of tangential coupled stress in all three theories $\mathrm{CD}, \mathrm{L}-\mathrm{S}$ and G-L for $\alpha=1$ are large compared to those for $\alpha=1.8$ in the ranges $0 \leq x \leq 3.5$ and $4.5 \leq x \leq 6.2$ for $\mathrm{CD}$, in the ranges $0 \leq x \leq 3.3$ and $5.4 \leq x \leq 7.7$ for $\mathrm{L}-\mathrm{S}$ and in the ranges $0 \leq x \leq 3.6$ and $6.2 \leq x \leq 8.7$ for G-L, and small otherwise in the range $0 \leq x \leq 10$, while values are the same for the three theories and converge to zero at $x>10$.

Behaviors of normal stress $\sigma_{y y}$ in all three theories CD, L-S and G-L for $t=0.1$ are small compared to those for $t=0.4$ in the ranges $0 \leq x \leq 3.7$ and $6 \leq x \leq 7.8$ for $\mathrm{CD}$, in the ranges $0 \leq x \leq 4.5$ and $6.9 \leq x \leq 8.5$ for L-S and in the ranges $0 \leq x \leq 5.1$ and $7.6 \leq x \leq 10$ for G-L, but in the ranges $3.7 \leq x \leq 6$ and $7.8 \leq x \leq 10$ for $\mathrm{CD}$, in the range $4.5 \leq x \leq 6.9$ for L-S and in the range $5.1 \leq x \leq 7.6$ for $\mathrm{G}-\mathrm{L}$ are large, while the values are the same for the three theories at $x>10$, as depicted in Fig. 11.

Figure 12 shows that the behavior of normal stress $\sigma_{y y}$ in all three theories CD, L-S and G-L for the two different values of magnetic field (i.e. $\alpha=1$ without magnetic field and $\alpha=1.8$ with magnetic field) is similar as a wave. The values of normal stress $\sigma_{y y}$ for $\alpha=1.8$ are large compared to those for $\alpha=1$ in the ranges $0 \leq x \leq 3.2$ and $4.8 \leq x \leq 6.9$ for $\mathrm{CD}$, in the ranges $0 \leq x \leq 3.5$ and $5.5 \leq x \leq 8.4$ for $\mathrm{L}-\mathrm{S}$ and in the ranges $0 \leq x \leq 3.9$ and $6.2 \leq x \leq 9$ for G-L, but these values are small in the ranges $3.2 \leq x \leq 4.8$ and $6.9 \leq x \leq 8.3$ for $\mathrm{CD}$, in the ranges $3.5 \leq x \leq 5.5$ and $5.5 \leq x \leq 8.4$ for $\mathrm{L}-\mathrm{S}$ and in the range $3.9 \leq x \leq 6.2$ for $\mathrm{G}-\mathrm{L}$, while the values are the same for the three theories at $x>10$ and converge to zero.

By comparing the figures of solutions obtained under the three thermoelastic theories, important phenomena are observed:

1. The curves in the context of the CD, L-S and G-L theories decrease exponentially with increasing $x$, this indicates that the thermoelastic waves are unattenuated and nondispersive, where purely thermoelastic waves undergo both attenuation and dispersion.

2. The values of the distributions of all the physical quantities converge to zero with increasing the distance $x$.

3. The curves of the physical quantities with L-S theory in most of figures are lower in comparison 
with those under G-L theory, due to the relaxation times.

4. The dependence of the internal heat source has a significant effect on the thermal and mechanical interactions, and plays a significant role in all the physical quantities.

5. It is clear from Figs. 2, 4, 6, 8, 10, and 12 that the magnetic field plays a significant role in all the physical quantities.

6. It is clear from Figs. 1, 3, 5, 7, 9, and 11 that the different times play a significant role in all the physical quantities.

7. All the physical quantities satisfy the boundary conditions and initial conditions.

\section{Conclusion}

Transient waves created by a line heat source inside isotropic homogeneous thermoelastic half-space permeating into a uniform magnetic field are studied under three theories of generalized thermoelasticity CD, L-S and G-L. The problem is reduced to the solution of three equations, one involving the elastic vector potential and the other two coupled, involving the thermoelastic scalar potential and the temperature. The normal mode analysis used in this article to solve the problem is applicable to a wide range of problems in different branches (Othman [20], Othman and Song [21] and Othman and Singh [22]). It can be applied to boundary-layer problems, which are described by the linearized Navier-Stokes equations in electrohydrodynamics (Othman [23]). This method gives exact solutions without any assumed restrictions on either the temperature or stress distributions. The results are graphically described for the medium of copper. We can conclude that the magnetic field has a great effect on the displacement components and this effect produces the same trend under the three theories. The results show that the relaxation times have salient effect to the distribution of displacement at small values of time. The present theoretical results may provide interesting information for experimental scientists/researchers/seismologist working on this subject.

\section{References}

[1] H.W. Lord, Y. Shulman, J. Mech. Phys. Solids 15, 299 (1967).

[2] P. Puri, Int. J. Eng. Sci. 10, 467 (1972).

[3] A. Nayfeh, S. Nemat-Nasser, ZAMP 23, 52 (1972).

[4] V.K. Agarwal, Acta Mech. 31, 185 (1979).

[5] M. Ezzat, M.I.A. Othman, J. Thermal Stresses 25, 409 (2002).

[6] H.H. Sherief, K.A. Helmy, Int. J. Eng. Sci. 40, 587 (2002).

[7] M.I.A. Othman, J. Thermal Stresses 25, 1027 (2002).

[8] M. Ezzat, M.I.A. Othman, A.S. El-Karamany, J. Thermal Stresses 24, 411 (2001).

[9] A.E. Green, K.E. Lindsay, J. Elasticity 2, 1 (1972).

[10] V.K. Agarwal, Acta Mech. 34, 181 (1979).

[11] M. Schoenberg, D. Censor, Quart. Appl. Math. 31, 115 (1973).

[12] S.K. Roy Choudhuri, S. Mukhopadhyay, Int. J. Math. Math. Sci. 23, 479 (2000).

[13] M.A. Ezzat, M.I.A. Othman, Int. J. Eng. Sci. 38, 107 (2000).

[14] R. Dhaliwal, J. Rokne, J. Thermal Stresses 12, 259 (1989).

[15] M.I.A. Othman, Int. J. Solids Structures 41, 2939 (2004).

[16] S. Chakravorty, A. Chakravorty, Int. J. Math. Math. Sci. 21, 595 (1998).

[17] D.S. Chandrasekharaiah, K.S. Srinath, J. Elasticity 50, 97 (1998).

[18] D.S. Chandrasekharaiah, H.N. Murthy, Acta Mech. 89, 1 (1991).

[19] R.B. Hetnarski, J. Ignaczak, J. Thermal Stresses 16 , 473 (1993).

[20] M.I.A. Othman, Acta Mech. 174, 129 (2005).

[21] M.I.A. Othman, Y. Song, Appl. Math. Mod. 32, 811 (2008).

[22] M.I.A. Othman, B. Singh, Int. J. Solids Structures 44, 2748 (2007).

[23] M.I.A. Othman, ZAMP 55, 468 (2004). 